\title{
АНАЛИТИЧЕСКОЕ КОНСТРУИРОВАНИЕ ОПТИМАЛЬНЫХ СИСТЕМ УПРАВЛЕНИЯ ДВИЖЕНИЕМ ЛИНЕЙНОГО ОБЪЕКТА ПО ЗАДАННОЙ ТРАЕКТОРИИ ПРИ СТОХАСТИЧЕСКИХ ВОЗДЕЙСТВИЯХ
}

\author{
Блохин Л.Н. ${ }^{1}$, д.т.н., проф., Осадчий С.И. ${ }^{2}$, д.т.н., проф., Калич В.М. ${ }^{2}$, к.т.н., проф., Кочерженко Ю.М. ${ }^{2}$ \\ ${ }^{1}$ ORCID: 0000-0002-1811-3594 \\ ${ }^{2}$ ORCID: $\underline{0000-0002-9907-5496}$ \\ ${ }^{1}$ Национальный авиационный университет, г. Киев \\ ${ }^{2}$ Кировоградский национальный технический университет, г. Кировоград, E-mail: $\underline{\operatorname{srg} 2005 @ \mathrm{ukr} . n e t}$
}

Copyright (C) 2014 by author and the journal "Automation technological and business - processes".

This work is licensed under the Creative Commons Attribution International License (CC BY).

http://creativecommons.org/licenses/by/4.0/

\section{Анотація}

Работа посвящена разработке нового метода расчета матриц передаточных функций трактов управления оптимального многомерного регулятора. Регулятор предназначен для максимизации точности перехода линейного многомерного объекта управления из одного установившегося режима в другой. Предполагается что на объект управления действуют возмущения, а датчики измерительной информации обладают инерцией и помехами. Как возмущения, так и помехи представляют собой аддитивную комбинацию регулярных и случайных составляющих. Случайные составляющие ограничены классом взаимосвязанных стационарных процессов с дробно-рациональными матрицами спектральных плотностей. В основу разработки метода положена постановка задачи синтеза с использованием новой структурной схемы системы управления, полученной с учетом результатов аттестации динамики датчиков. Синтез регулятора осуществляется в частотной области методом Винера-Колмогорова. Полученный в результате решения задачи синтеза новый алгоритм позволяет найти матрицы передаточных функций регулятора, которые обеспечивают минимум соответствующих квадратичных критериев качества. Первый из них представляет сумму определенным образом взвешенных интегральных квадратичных отклонений регулярных ошибки следования объекта по траектории и сигнала управления. Второй критерий представляет сумму взвешенных дисперсий случайных составляющих ошибки и сигнала управления. Для ипользования предложенного алгоритма необходимо выполнить операции винеровской факторизации и сепарации дробно-рациональных матриц. Соответствующие функции содержатся в свободно распространяемом пакете программ SciLab.

\section{Abstract}

This article is devoted to the development of a new method of calculating transfer functions matrices of optimal multivariable regulator's control paths. The regulator is designed to maximize the accuracy of the linear multivarible control object transition from one steady state to another. It is assumed that control object acting disturbances and sensors measuring data have inertia and noise. Both disturbances and noises are an additive combination of regular and random components. Random components belong to a class of interconnected stationary processes with rational spectral density matrices. New method development is based on formulation of a synthesis problem with the help of a new control system block diagram, which is obtained as a result of taking into account sensors dynamics certification data. 
Synthesis of the regulator is carried out in the frequency domain by the Wiener-Kolmogorov method. A new algorithm, which is obtained as a result of synthesis problem solution, allows you to find the matrix of regulator transfer functions , which provide a minimum of corresponding quadratic quality criteria. The first of them is equal to the sum of certain way weighted squared deviations regular repetition errors of the object path and control signals. The second criterion is equal to the sum of the weighted variance of the random error components and the control signals. To execute the proposed algorithm it is necessary to perform the operations of Wiener factorization and separation of rational matrices. The corresponding functions are contained in the freely distributed software package SciLab.

Ключовые слова

Управление, объект, движение, матрица, факторизация, сепарация, минимум, траектория

Keywords

Control, object, movement, matrix, factorization, separation, minimum, trajectory

Повышение точности следования подвижного объекта по заданной траектории или смены режима технологического процесса в условиях действия возмущений и помех связано с разработкой и использованием оптимальных систем управления [1]. Аналитическое конструирование подобных систем [2] - наиболее эффективный путь их создания. Основная проблема, которая встречается на таком пути, не смотря на многообразие постановок задач, методов, приемов и средств их решения [3,4,5], связана с необходимостью учета совместного действия случайной и регулярной составляющих стохастических воздействий на линеаризованый объект управления при синтезе системы упраления.

Решение этой проблемы на основе теоремы разделения [4] предполагает, что случайные возмущения и помехи, действующие на объект управления, являются «белым шумом». В реальных условиях функционирования технологического объекта, летательного аппарата, морского судна, суперкавитационного объекта, подвижного робота это условие не выполняется. Учет особенностей динамики возмущений и помех в пространстве состояний часто приводит к возникновению эффекта «проклятья» размерности.

Известные методы синтеза оптимальных многомерных систем управления в частотной области, обзор которых приведен, например, в работах [5,6,7], свободны от ограничения на класс моделей стационарных случайных воздействий. В тоже время, эти методы позволяют находить оптимальные многомерные регуляторы для случаев работы системы либо при случайных воздействиях, либо при регулярных.

Цель

Целью данной работы является разработка алгоритма синтеза оптимальных матриц передаточных функций регулятора в системе программного управления линейным многомерным объектом управления при переходе из одного установившегося режима в другой при стохастических воздействиях ( аддитивной смеси случайного стационарного процесса с детерминированным сигналом).

Пусть заданная траектория перехода состоит из двух частей (зон) (рис. 1$)$ : спрямленной наклонной ветви $l_{0}$ и ее горизонтального продолжения $r_{0}$. Траектория расположена в координатной плоскости Ozt, a ось времени $\mathrm{t}$

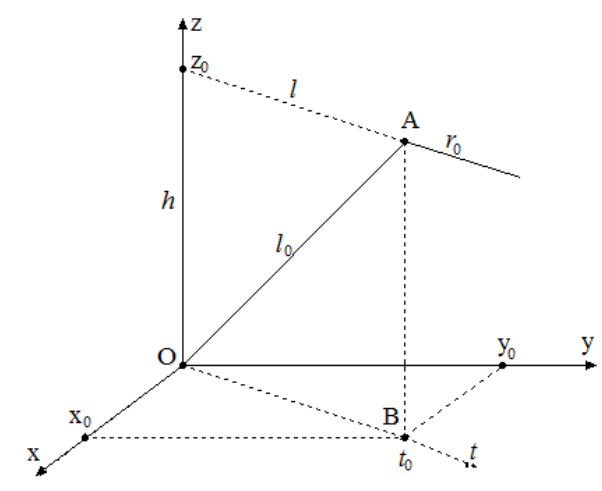

Рис. 1 - Расположение заданной траектории движения объекта в выбранной системе координат 


\section{МЕТОДИ ТА АЛГОРИТМИ ЕФЕКТИВНОГО УПРАВЛІННЯ ОБ‘ЭКТАМИ}

совпадает с биссектрисой угла хОу. Для определенности пусть координаты точки А относительно системы координат Охуz определяются соотношением

$$
\left[\begin{array}{l}
x_{0} \\
y_{0} \\
z_{0}
\end{array}\right]=\left[\begin{array}{c}
141.25 \\
141.25 \\
10
\end{array}\right], \quad \kappa M,
$$

тогда длина ветви $l_{0}$ равна 200 км, а ее проекция на горизонтальную плоскость $l$ составляет 199.75 км. Будем полагать также, что время движения объекта по наклонной ветви должно $t_{0}$ должно быть равно $100 \mathrm{c}$.

Принимается, что на наклонном участке траектории объект должен двигаться вдоль траектории с постоянной скоростью, вектор которой $V_{1}$ равен

$$
V_{1}=\left[\begin{array}{c}
V_{x} \\
V_{y} \\
V_{z}
\end{array}\right],
$$

где $V_{\mathrm{x}}, V_{\mathrm{y}}, V_{\mathrm{z}}$ - проекции вектора $V_{1}$ на оси системы координат Охуz, а на горизонтальном участке - с такой постоянной скоростью $V_{2}$, что

$$
V_{2}=\left[\begin{array}{c}
V_{x} \\
V_{y} \\
0
\end{array}\right] .
$$

В таком случае, изображение Лапласа вектора перемещения центра масс объекта вдоль наклонной части траектории при нулевых начальных условиях следует представить в виде

$$
\xi_{1}(s)=\frac{1}{s} V_{1},
$$

а изменение во времени вектора координат центра масс объекта при движении по горизонтальному участку траектории с учетом (рис.1) определить уравнением

$$
\xi_{2}(t)=V_{2} t+\left[\begin{array}{c}
0 \\
0 \\
z_{0}
\end{array}\right] 1\left(t-t_{0}\right)
$$

где $1\left(t-t_{0}\right)$ - единичная ступенчатая функция с задержкой на время движения объекта по наклонной траектории $t_{0}$

$$
1\left(t-t_{0}\right)= \begin{cases}0 & \forall t<t_{0} \\ 1 & \forall t \geq t_{0}\end{cases}
$$

Изображение вектора сигналов (1) с учетом принятых допущений представляется так

$$
\xi_{2}(s)=\frac{1}{s^{2}}\left[\begin{array}{c}
V_{x} \\
V_{y} \\
s z_{0} e^{-100 s}
\end{array}\right] .
$$


Перемещение объекта из первой зоны во вторую должно производиться по специальному командному переключению. Поскольку частотные характеристики векторов координат $\xi_{1}$ и $\xi_{2}$ на каждом участке траектории полагаются неизменными, то и алгоритмы синтеза оптимальных систем управления движением по этим участках принципиально одинаковы по структуре. Для исключения повторов в дальнейшем изложении задач синтеза изображения векторов входных сигналов $\xi_{1}(\mathrm{~s})$ и $\xi_{2}(\mathrm{~s})$ будут обозначены как $\xi(\mathrm{s})$.

Замкнутая система управления, обеспечивающая движение объекта по заданной траектории (рис. 1), включает как минимум два тракта: задания программы движения (рис. 2а) и измерения выходных сигналов (рис. 2б).

Тракт задания программы предназначен для наблюдения за вектором $\xi$ и формирования вектора оценок программы движения $\xi$. Наблюдение происходит с помощью задающего устройства с матрицей передаточных функций $K_{0}(\mathrm{~s})$ и сопровождается аддитивными помехами, представленными на схеме рис. $2 \mathrm{a}$ вектором $\varphi_{0}$. Формирование вектора оценок $\tilde{\xi}$ осуществляется путем компенсации влияния помех измерения вектора $y_{0}$ с помощью вектора оценок погрешностей задания программы $\tilde{\varphi}_{\text {o }}$, полученного экспериментально.

Тракт измерения выходных сигналов (рис. 2б) предназначен для формирования вектора оценок выходных сигналов объекта управления $\tilde{x}$ по результатам измерения вектора $x$ с помощью измерителей, динамику которых характеризует матрица передаточных функций $K_{2}(\mathrm{~s})$, а шум измерения - вектор $\varphi_{2}$. Вектор $\tilde{\varphi}_{2}$ представляет погрешности измерения, полученные при аттестации характеристик измерителей.

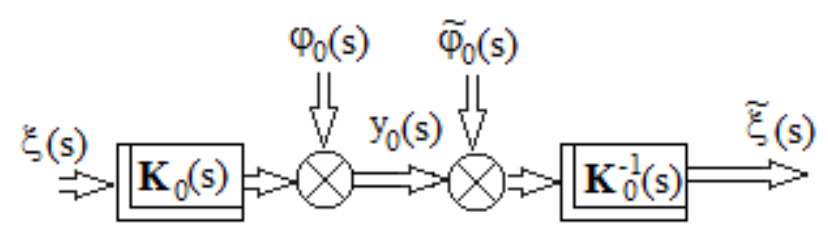

a)

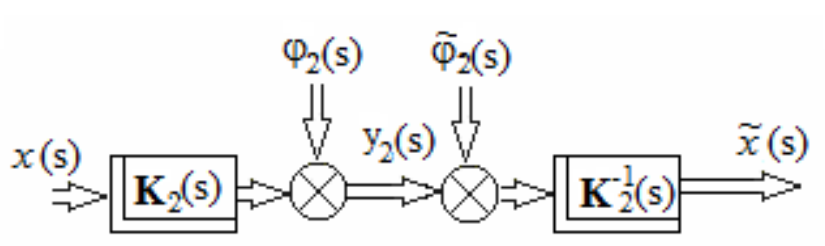

б)

Рис. 2 - Структурные схемы трактов задания прграммы движения (а) и измерения выходных сигналов (б)

На рис. 3 приведены структурные схемы системы управления траекторным движением объекта управления при детерминированных(рис. 3а) и случайных (рис. 3б) воздействиях. Различный характер воздействий на систему подчеркивается нижними индексами «d» и «s» при идентификаторах векторов (идекс «d» обозначает детерминированный сигнал, индекс «s» - центрированный стационарный случайный). Для краткости записи комплексную переменную $s=$ ј

Движение объекта управления, представленного на структурной схеме (рис. 3a), описывается системой линейных дифференциальных уравнений, преобразованной по Фурье, вида

$$
P x_{d}=M u_{d}+\Psi_{d} g
$$

а движение объекта управления, представленного на рис. 3б, характеризует система уравнений

$$
P x_{s}=M u_{s}+\Psi_{s} \Delta \text {, }
$$

где $P$ - заданная матрица размерности $n \times n$, элементы которой -полиномы от комплексной переменной $s ; x_{\mathrm{d}}-\mathrm{n}-\mathrm{mepный}$ вектор детерминированных составляющих выходных координат объекта; $M$ - полиномиальная матрица размерности $n \times m ; u_{\mathrm{d}}-\mathrm{m}$-мерный вектор детерминированных сигналов управления; $\Psi_{\mathrm{d}}$ - вектор комплексных коэффициентов передачи фильтра, формирующего вектор сигналов детерминированного возмущения из единичной ступенчатой функции $g$; $\Psi_{\mathrm{s}}$ - матрица передаточных функций формирующего фильтра вектора стационарных случайных возмущений; $\Delta$ - белый шум. Структурные схемы системы управления включают также матрицу передаточных функций задатчиков программы движения $K_{l}$; матрицы передаточных функций регуляторов трактов детерминированных и случайных сигналов $W_{\mathrm{d}}$ и $W_{\mathrm{s}}$, которые необходимо найти.

Итак, общее движение объекта управления (рис. За, 3б) на основе принципа суперпозиции следует описать системой обыкновенных дифференциальных уравнений, преобразованных по Фурье при нулевых начальных условия, вида

$$
P x=M u+\Psi_{d} g+\Psi_{s} \Delta,
$$

где $x$ - результирующий n-мерный вектор выходных координат объекта

$$
x=x_{d}+x_{s}
$$

$u$ - результирующий m-мерный вектор сигналов управления такой, что 


\section{МЕТОДИ ТА АЛГОРИТМИ ЕФЕКТИВНОГО УПРАВЛІННЯ ОБ‘ЭКТАМИ}

$$
u=u_{d}+u_{s}
$$

Вектор оценок выходных сигналов $\tilde{x}$ с учетом структурных схем (рис. 2, рис. 3) определяется уравнением

$$
\widetilde{x}=x+K_{2}^{-1} \widetilde{\zeta}_{2},
$$

в котором $\tilde{\zeta}_{2}$ - вектор отклонений действительных шумов измерения от оценок шумов, полученных при метрологической аттестации измерителей,
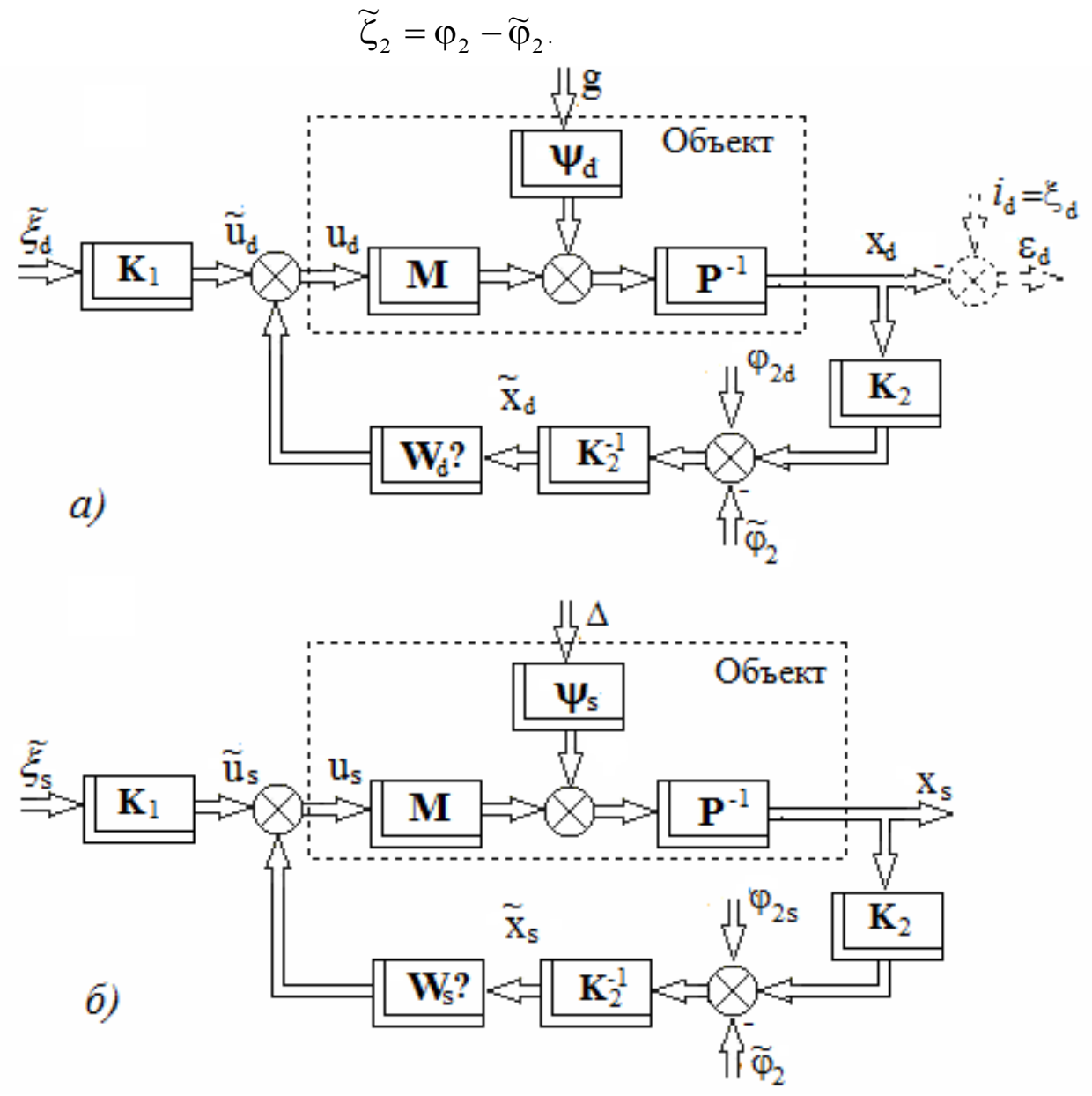

Рис. 3 - Структурные схемы системы управления траекторным движением объекта при регулярных а) и случайных б) воздействиях

Как показано на рис. За изображение детерминированного вектора сигналов ошибок управления траекторным движением объекта может быть представлено так

$$
\varepsilon_{d}=x_{d}-\xi_{d}
$$

Если ввести в рассмотрение приведенные к одному входу векторы детерминированных $\eta_{\mathrm{d}}$ и случайных $\eta_{\mathrm{s}}$ обобщенных возмущающих воздействий, которые равны

$$
\begin{aligned}
& \eta_{d}=M K_{1} \widetilde{\xi}_{d}+\Psi_{d} g+P K_{2}^{-1} \widetilde{\zeta}_{2 d}, \\
& \eta_{s}=M K_{1} \widetilde{\xi}_{s}+\Psi_{s} \Delta+P K_{2}^{-1} \widetilde{\zeta}_{2 s},
\end{aligned}
$$

а также определить векторы изображений регулярной и случайной составляющих управляющих воздействий в таком виде

$$
u_{d}=F_{u d} \eta_{d} ; u_{s}=F_{u s} \eta_{s}
$$


где $F_{u d}$ - матрица передаточных функций размерности $m \times n$ от входа $\eta_{\mathrm{d}}$ к вектору $u_{\mathrm{d}}$ замкнутой системы управления, представленной на рис. $4 ; F_{\text {us }}$ - матрица передаточных функций размерности $m \times n$ от входа $\eta_{\mathrm{s}}$ к вектору $u_{\mathrm{s}}$ этой же системы управления, то структурные схемы на рис. 3 можно преобразовать к единой системе управления, имеющей два тракта: регулярных сигналов и случайных сигналов (рис.4).

Для сокращения в дальнейшем записи выражений (4) и (5) целесообразно ввести следующие обозначения

$$
\Phi=P^{-1} M ; f_{s}=P^{-1} \Psi_{s} ; f_{d}=P^{-1} \Psi_{d} .
$$

Учитывая соотношения (5) и (6), уравнение для вектора случайных составляющих ошибки

$$
\varepsilon_{s}=\Phi \mathrm{F}_{\mathrm{us}} \eta_{s}+\Phi \mathrm{K}_{1} \widetilde{\xi}_{s}+f_{s} \Delta
$$

и воспользовавшись теоремой Винера-Хинчина, возможно составить транспонированные матрицы спектральных плотностей векторов сигналов $\varepsilon_{\mathrm{s}}, \eta_{\mathrm{s}}, u_{\mathrm{s}}$ при условии независимости векторов программных сигналов и возмущений

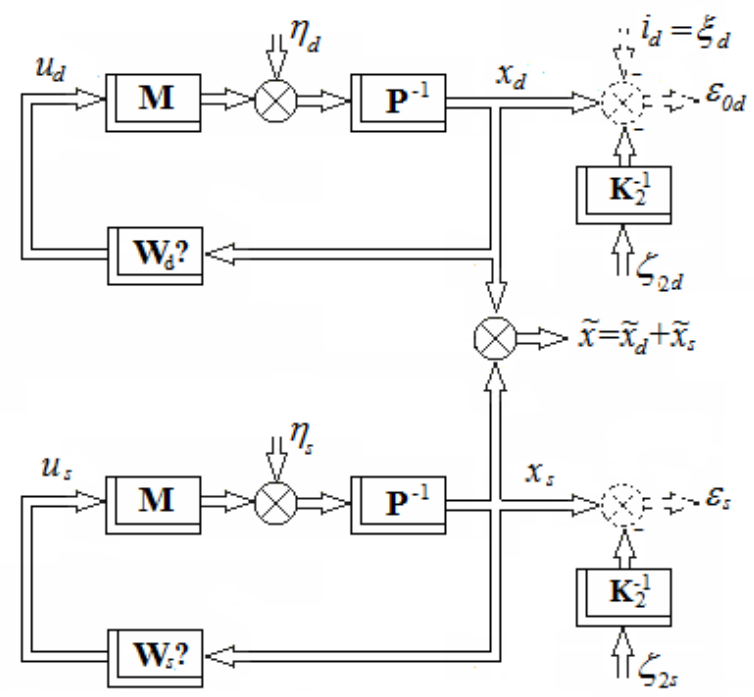

Рис. 4 - Преобразованная структурная схема системы управления траекторным движением объекта

$$
\begin{gathered}
S_{\varepsilon \varepsilon}^{\prime}=\Phi F_{u s} S_{\eta \eta}^{\prime} F_{u s^{*}} \Phi_{*}+\Phi F_{u s}\left[M K_{1} S_{\xi \xi}^{\prime} K_{1^{*}} \Phi_{*}+\frac{\sigma_{\Delta}^{2}}{\pi} \Psi_{s} f_{s^{*}}\right]+\left[\Phi K_{1} S_{\xi \xi}^{\prime} K_{1^{*}} M_{*}+\frac{\sigma_{\Delta}^{2}}{\pi} f_{s} \Psi_{s^{*}}\right] F_{u s^{*}} \Phi_{*}+ \\
+\Phi K_{1} S_{\xi \xi}^{\prime} K_{1^{*}} \Phi_{*}+\frac{\sigma_{\Delta}^{2}}{\pi} f_{s} f_{s^{*}}, \\
S_{\eta \eta}^{\prime}=M K_{1} S_{\xi \xi}^{\prime} K_{1^{*}} M_{*}+\frac{\sigma_{\Delta}^{2}}{\pi} \Psi_{s} \Psi_{s^{*}}+P K_{2}^{-1} S_{\zeta \zeta}^{\prime} K_{2^{*}}^{-1} P_{*}, \\
S_{u u}^{\prime}=F_{u s} S_{\eta \eta}^{\prime} F_{u s^{*}}
\end{gathered}
$$

где $S_{\xi \xi}^{\prime}$ - транспонированная матрица спектральных плотностей случайной составляющей вектора оценок программных сигналов $\xi_{s} ; S_{\zeta \zeta}^{\prime}$ - транспонированная матрица спектральных плотностей случайных составляющих вектора отклонений действительных шумов измерения от оценок шумов $\zeta_{2 s}$; нижний индекс * знак эрмитового сопряжения матрицы.

Качество системы управления траекторным движением (рис.4) целесообразно оценивать с помощью двух функционалов, характеризующих ее работу при детерминированных и случайных воздействиях. Функционал качества при детерминированных воздействиях принят в виде

$$
I=\frac{1}{2 \pi j} \int_{-j \infty}^{j \infty} \operatorname{tr}\left(\varepsilon_{0 d} \varepsilon_{0 d^{*}} R_{d}+u_{d} u_{d^{*}} C_{d}\right) d s,
$$


а функционал качества системы при случайных воздействиях определен так

$$
e=\frac{1}{j} \int_{-j \infty}^{j \infty} \operatorname{tr}\left(S_{\varepsilon \varepsilon}^{\prime} R_{s}+S_{u u}^{\prime} C_{s}\right) d s,
$$

где $t r$ - знак операции поиска следа матрицы, $R_{\mathrm{d}}, C_{\mathrm{d}}, R_{\mathrm{s}}, C_{\mathrm{s}}$ - весовые матрицы, $j$ - комплексная единица.

По аналогии с работой [7] введены в рассмотрение матрицы передаточных функций $F_{\mathrm{xd}}, F_{\mathrm{xs}}$ от векторов обобщённых возмущений $\eta_{\mathrm{d}}, \eta_{\mathrm{s}}$ к векторам выходных сигналов $x_{\mathrm{d}}, x_{\mathrm{s}}$ связанные с матрицами передаточных функций $F_{u d}, F_{\text {us }}$ следующими уравнениями

$$
F_{x d}=\Phi F_{u d}+P^{-1} ; F_{x s}=\Phi F_{u s}+P^{-1}
$$

Задача аналитического конструирования состоит в том, чтобы по известным матрицам $P, M, K_{1}, K_{2}, \Psi_{\mathrm{d}}, \Psi_{\mathrm{s}}, S_{\xi \xi}, S_{\zeta \zeta}$ найти матрицы передаточных функций регуляторов $W_{\mathrm{d}}$ и $W_{\mathrm{s}}$, включение который в цепи обратных связей обеспечит устойчивость системы и доставит минимум функционалам качества (10), (11).

Решение задачи предлагается искать в два этапа. Вначале необходимо осуществить аналитическое конструирование структуры матрицы передаточных функций регулятора $W_{\mathrm{d}}$, работающего при детерминированных воздействиях. Для этого, учитывая уравнения (2)-(5), изображение вектора сигналов детерминированной ошибки представлено в виде

$$
\varepsilon_{0 d}=\Phi \mathrm{F}_{\mathrm{ud}} \eta_{d}-\left[E_{n}-\Phi K_{1}\right] \xi_{d}+f_{d} g
$$

где $E_{\mathrm{n}}-$ единичная матрица размерности $n \times n$

Подстановка вектора (13) в функционал (10) позволяет представить его как

$$
\begin{gathered}
I=\frac{1}{2 \pi j} \int_{-j \infty}^{j \infty} \operatorname{tr}\left\{\left[\Phi F_{u d} \eta_{d} \eta_{d^{*}} F_{u d^{*}} \Phi_{*}-\Phi F_{u d} \eta_{d} \xi_{d^{*}}\left(E_{n}-K_{1^{*}} \Phi_{*}\right)+\Phi F_{u d} \eta_{d} g_{*} f_{d^{*}}+f_{d} g \eta_{d^{*}} F_{u d^{*}} \Phi_{*}-\right.\right. \\
-\left(E_{n}-\Phi K_{1}\right) \xi_{d} \eta_{d^{*}} F_{u d^{*}} \Phi_{*}+\left(E_{n}-\Phi K_{1}\right) \xi_{d} \xi_{d^{*}}\left(E_{n}-K_{1^{*}} \Phi_{*}\right)-\left(E_{n}-\Phi K_{1}\right) \xi_{d} g_{*} f_{d^{*}}- \\
\left.\left.\quad-f_{d} g \xi_{d^{*}}\left(E_{n}-K_{1^{*}} \Phi_{*}\right)+f_{d} g g_{*} f_{d^{*}}\right] R_{d}+F_{u d} \eta_{d} \eta_{d^{*}} F_{u d^{*}} C\right\} d s .
\end{gathered}
$$

Задача поиска матрицы передаточных функций $W_{\mathrm{d}}$ решается [7] путем минимизации функционала (14) с использованием идеи метода Винера-Колмогорова на классе устойчивых и физически реализуемых матриц

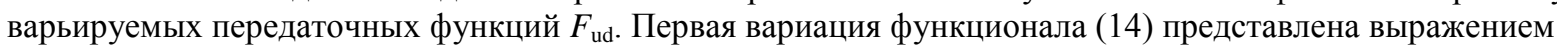

$$
\begin{aligned}
& \delta I=\frac{1}{2 \pi j} \int_{-j \infty}^{j \infty} \operatorname{tr}\left\{\left[\left(\Phi_{*} R_{d} \Phi+C_{d}\right) F_{u d} \eta_{d} \eta_{d^{*}}+\Phi_{*} R_{d} f_{d} g \eta_{d^{*}}-\Phi_{*} R_{d}\left(E_{n}-\Phi K_{1}\right) \xi_{d} \eta_{d^{*}}\right] \delta F_{u d^{*}}+\right. \\
& \left.\quad+\delta F_{u d}\left[\eta_{d} \eta_{d^{*}} F_{u d^{*}}\left(C_{d}+\Phi_{*} R_{d} \Phi\right)+\eta_{d} g_{*} f_{d^{*}} R_{d} \Phi-\eta_{d^{*}} \xi_{d}\left(E_{n}-K_{1^{*}} \Phi_{*}\right) R_{d} \Phi\right]\right\} d s .
\end{aligned}
$$

Для поиска матрицы варьируемых передаточных функций, указанного класса, необходимо ввести следующие обозначения

$$
\begin{gathered}
\Gamma_{d^{*}} \Gamma_{d}=\Phi_{*} R_{d} \Phi+C_{d} ; \widetilde{D}_{d} J \widetilde{D}_{d^{*}}=\eta_{d} \eta_{d^{*}} \\
T_{0}+T_{+}+T_{-}=\Gamma_{d^{*}}^{-1} \Phi_{*} R_{d}\left[f_{d} g-\left(E_{n}-\Phi K_{1}\right) \xi_{d}\right] \eta_{d^{*}} \widetilde{D}_{d^{*}}^{-1} J^{-1}
\end{gathered}
$$

где $\Gamma_{\mathrm{d}}$ - аналитическая вместе с обратной в правой полуплоскости (ППП) комплексного аргумента $s$ дробнорациональная матрица, найденная в результате факторизации [8] суммы в правой части первого уравнения; $\tilde{D}_{\mathrm{d}}-$ аналитическая в ППП дробно-рациональная матрица, являющаяся результатом Ј-спектральной факторизации [9] произведения векторов $\eta_{\mathrm{d}} \eta_{\mathrm{d} *}$; индексы $0,+,-$-обозначают результаты сепарации правой части последнего выражения. С учетом принятых обозначений (16) вариация (15) приводится к виду

$$
\begin{aligned}
\delta I= & \frac{1}{2 \pi j} \int_{-j \infty}^{j \infty} \operatorname{tr}\left[\Gamma_{d^{*}}\left(\Gamma_{d} F_{u d} \widetilde{D}_{d}+T_{0}+T_{+}+T_{-}\right) J \widetilde{D}_{d^{*}} \delta F_{u d^{*}}+\right. \\
& \left.+\delta F_{u d} \widetilde{D}_{d} J\left(\widetilde{D}_{d^{*}} F_{u d^{*}} \Gamma_{d^{*}}+T_{0^{*}}+T_{+^{*}}+T_{-^{*}}\right) \Gamma_{d}\right] d s,
\end{aligned}
$$

а условие приближенного равенства нулю вариации $(17)$ на классе устойчивых и физически реализуемых матриц $F_{\text {ud }}$ будет таким

$$
\Gamma_{d} F_{u d} \widetilde{D}_{d}=-\left(T_{0}+T_{+}\right)
$$


Алгоритм синтеза оптимизированной структуры матрицы $F_{u d}$ в этом варианте задачи должен быть таким

$$
F_{u d}=-\Gamma_{d}^{-1}\left(T_{0}+T_{+}\right) \widetilde{D}_{d}^{-1}
$$

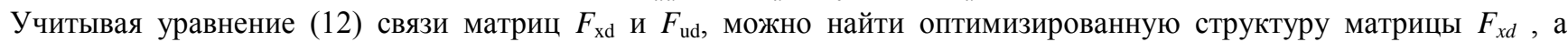
оптимизированную структуру матрицы передаточных функций регулятора в тракте детерминированных сигналов $W_{d}$ можно представить в виде

$$
W_{d}=F_{u d}\left(\Phi F_{u d}+P^{-1}\right)^{-1}
$$

Решение поставленной задачи синтеза (19) является приближенным, поскольку числовая матрица $J$, найденная при $J$-спектральной факторизации является особенной.

Точное решение поставленной задачи возможно получить, если все особенности модели динамики вектора $\eta_{\mathrm{d}}$ находятся в левой полуплоскости комплексного переменного $s=j \omega$. Учитывая высказанное предположение, вариацию (15) можно переписать так

$$
\begin{aligned}
\delta I=\frac{1}{2 \pi j} \int_{-j \infty}^{j \infty} \operatorname{tr}\left\{\left[\left(\Phi_{*} R_{d} \Phi+C_{d}\right) F_{u d} \eta_{d}+\Phi_{*} R_{d} f_{d} g-\Phi_{*} R_{d}\left(E_{n}-\Phi K_{1}\right) \xi_{d}\right] \eta_{d^{*}} \delta F_{u d^{*}}+\right. \\
\left.+\delta F_{u d} \eta_{d}\left[\eta_{d^{*}} F_{u d^{*}}\left(C_{d}+\Phi_{*} R_{d} \Phi\right)+g_{*} f_{d^{*}} R_{d} \Phi-\xi_{d}\left(E_{n}-K_{1^{*}} \Phi_{*}\right) R_{d} \Phi\right]\right\} d s
\end{aligned}
$$

Как и ранее введем следующие обозначения

$$
\Gamma_{d^{*}} \Gamma_{d}=\Phi_{*} R_{d} \Phi+C_{d} ; T_{0}+T_{+}+T_{-}=\Gamma_{d^{*}}^{-1} \Phi_{*} R_{d}\left[f_{d} g-\left(E_{n}-\Phi K_{1}\right) \xi_{d}\right],
$$

тогда уравнение для расчета физически реализуемой варьируемой матрицы $F_{\mathrm{d}}$ приобретает следующий вид

$$
F_{u d}=-\Gamma_{d}^{-1}\left(T_{0}+T_{+}\right) \eta_{d}^{\#},
$$

где \# - знак псевдообращения вектора.

По Гантмахеру [10] справедливо равенство

где * - символ транспонирования;

$$
\eta_{d}^{\#}=C^{*}\left(C C^{*}\right)^{-1}\left(B^{*} B\right)^{-1} B^{*}
$$

$$
B=\eta_{d} ; C=\left[\begin{array}{ll}
1 & 0
\end{array}\right] ; C C_{*}=1 ; B^{*} B=\sum_{j=1}^{3} \eta_{d j}^{2}
$$

Подстановка данных (23) в уравнение (22) позволяет найти выражение для расчета псевдообратного вектора

$$
\eta_{d}^{\#}=\frac{\eta_{d}^{\prime}}{\sum_{k=1}^{3} \eta_{d k}^{2}}
$$

Таким образом выполнен первый этап решения задачи аналитического конструирования оптимальной системы управления движением линейного объекта при стохастических условиях.

Второй этап решения задачи конструирования посвящен обоснованию уравнения, необходимого для расчета матрицы передаточных функций регулятора $W_{\mathrm{s}}$ (рис. 4). В соответствии со структурными схемами системы управления (рис. 36 и рис. 4), выражениями (5) и (6), изображением вектора случайных составляющих сигнала ошибок $\varepsilon_{\mathrm{s}}$, а также соотношениями (7)-(9) функционал (11) возможно привести к виду

$$
e=\frac{1}{j} \int_{-j \infty}^{j \infty} \operatorname{tr}\left\{\left[\Phi F_{u s} S_{\eta \eta}^{\prime} F_{u s^{*}} \Phi_{*}+\Phi F_{u s} A+A_{*} F_{u s^{*}} \Phi+\Phi K_{1} S_{\xi \xi}^{\prime} K_{1^{*}} \Phi_{*}+\frac{\sigma_{\Delta}^{2}}{\pi} f_{s} f_{s^{*}}\right] R+F_{u s} S_{\eta \eta}^{\prime} F_{u s^{*}} C\right\} d s,
$$

где А - дробно-рациональная матрица, которая равна следующей сумме

$$
A=M K_{1} S_{\xi \xi}^{\prime} K_{1^{*}} \Phi+\frac{\sigma_{\Delta}^{2}}{\pi} \Psi_{s} f_{s^{*}} .
$$

Минимизацию функционала (24) на классе устойчивых и физически реализуемых матриц передаточных функций $F_{u s}$ необходимо выполнить методом Винера-Колмогорова [7]. Первая вариация функционала (24) имеет вид

$$
\delta e=\frac{1}{j} \int_{-j \infty}^{j \infty} \operatorname{tr}\left\{\left[\left(\Phi_{*} R \Phi+C\right) F_{u s} S_{\eta \eta}^{\prime}+\Phi_{*} R A_{*}\right] \delta F_{u s^{*}}+\delta F_{u s}\left[S_{\eta \eta}^{\prime} F_{u s^{*}}\left(\Phi_{*} R \Phi+C\right)+A R \Phi\right]\right\} d s .
$$


Если в выражение (25) внести следующие обозначения

$$
\Gamma_{s^{*}} \Gamma_{s}=\Phi_{*} R \Phi+C ; D_{s} D_{s^{*}}=S_{\eta \eta}^{\prime} ; T_{s}=T_{s 0}+T_{s+}+T_{s-}=\Gamma_{s^{*}}^{-1} \Phi_{*} R A_{*} D_{s^{*}}^{-1} ;
$$

то указанная вариация примет вид

$$
\delta e=\frac{1}{j} \int_{-j \infty}^{j \infty} \operatorname{tr}\left[\Gamma_{s^{*}}\left(\Gamma_{s} F_{u s} D_{s}+T_{s}\right) D_{s^{*}} \delta F_{u s^{*}}+\delta F_{u s} D_{s}\left(D_{s^{*}} F_{u s^{*}} \Gamma_{s^{*}}+T_{s^{*}}\right) \Gamma_{s}\right] d s .
$$

Тогда, условие равенства нулю вариации (27) на классе устойчивых и физически реализуемых варьируемых матриц $F_{\text {us }}$ будет таким

$\Gamma_{s} F_{u s} D_{s}=-\left(T_{s 0}+T_{s+}\right)$

а алгоритм синтеза оптимальной структуры варьируемой матрицы $F_{u s}$ имеет вид

$$
F_{u s}=-\Gamma_{s}^{-1}\left(T_{s 0}+T_{s+}\right) D_{s}^{-1} .
$$

Учитывая уравнение связи (12) между матрицами $F_{\text {us }}$ и $F_{\text {xs }}$ структура матрицы передаточных функций оптимального регулятора $W_{\mathrm{s}}$ может быть найдена из уравнения

$$
W_{s}=F_{u s}\left(\Phi F_{u s}+P^{-1}\right) .
$$

Таким образом, выполнен второй этап исследований, позволивший решить поставленную задачу аналитического конструирования.

В результате разработан практически реализуемый подход и алгоритмы синтеза оптимальных структур передаточных функций регуляторов, предназначенных для работы в трактах детерминированных и случайных сигналов системы управления траекторным движением лиейного многомерного объекта управления при стохастических воздействиях.

\section{Литература}

[1] Фельдбаум А.А. Основы теории оптимальных автоматических систем/ Фельдбаум А.А. - М.: Наука, 1966. 623c.;

[2] Летов А.М. Динамика полета и управление/ А.М.Летов - М.: Наука, 1969.- 360с.

[3] Методи сучасної теорії управління: Навч. посіб. / А.П. Ладанюк, В.Д. Кишенько, Н.М. Луцька, В.В. Іващук. К.: НУХТ, 2010. - 196c.;

[4] Тунік А.А. Основи сучасної теорії управління: Навч. посіб. / А.А. Тунік, О.О. Абрамович. - К.: НАУ, 2010. 260 c.;

[5] Ларин В.Б. Обобщенное уравнение Ляпунова и факторизация матричных полиномов/ В.Б.Ларин // Автоматика.- 1992.- №6.- С.3-8.;

[6] Осадчий С.И. Комбинированный метод синтеза оптимальных систем стабилизации многомерных подвижных объектов при стационарных случайных воздействиях / С.И. Осадчий, В.А. Зозуля. // Международный научнотехнический журнал «Проблемы управления и информатики». - 2013. - №3. - С. 40-49.;

[7] Азарсков В.Н. Методология конструирования оптимальных систем стохастической стабилизации: Монография / В.Н. Азарсков, Л.Н. Блохин, Л.С. Житецкий / Под ред. Блохина Л.Н. - К.: Книжное издательство НАУ, 2006. - 440с.;

[8] Davis M.C. Factoring the spectral matrix/ M.C.Davis // IEEE Trans. Automat. Cointr. - 1963.- AC-8, N 4. - p. 296305.;

[9] Kwakernaak H. Polynomial J-Spectral Factorization / H. Kwakernaak, M. Sebek // IEEE Transactions On Automatic Control - 1994 - VOL.39 - NO. 2 - pp. 315-328;

[10]Гантмахер Ф.Р. Теория матриц/ Ф.Р.Гантмахер -4-е изд.- М.: Наука, 1988.- 552c.

References

[1] Feldbaum A.A. Osnovyi teorii optimalnyih avtomaticheskih sistem/ Feldbaum A.A. - M.: Nauka, 1966. - 623s.

[2] Letov A.M. Dinamika poleta i upravlenie/ A.M.Letov - M.: Nauka, 1969.- 360s.;

[3] Metodi suchasnoYi teorIYi upravlInnya: Navch. posIb. / A.P. Ladanyuk, V.D. Kishenko, N.M. Lutska, V.V. Ivaschuk. - K.: NUHT, 2010. - 196s.;

[4] TunIk A.A. Osnovi suchasnoYi teorIYi upravlInnya: Navch. posIb. / A.A. TunIk, O.O. Abramovich. - K.: NAU, 2010. - 260 s.;

[5] Larin V.B. Obobschennoe uravnenie Lyapunova i faktorizatsiya matrichnyih polinomov/ V.B.Larin // Avtomatika.1992.- \#6.- S.3-8.; 
[6] Osadchiy S.I. Kombinirovannyiy metod sinteza optimalnyih sistem stabilizatsii mnogomernyih podvizhnyih ob'ektov pri statsionarnyih sluchaynyih vozdeystviyah / S.I. Osadchiy, V.A. Zozulya. // Mezhdunarodnyiy nauchnotehnicheskiy zhurnal «Problemyi upravleniya i informatiki». - 2013. - \#3. - S. 40-49.;

[7] Azarskov V.N. Metodologiya konstruirovaniya optimalnyih sistem stohasticheskoy stabilizatsii: Monografiya / V.N. Azarskov, L.N. Blohin, L.S. Zhitetskiy / Pod red. Blohina L.N. - K.: Knizhnoe izdatelstvo NAU, 2006. - 440s.;

[8] Davis M.C. Factoring the spectral matrix/ M.C.Davis // IEEE Trans. Automat. Cointr. - 1963.- AC-8, N 4. - p. 296305 ;

[9] Kwakernaak H. Polynomial J-Spectral Factorization / H. Kwakernaak, M. Sebek // IEEE Transactions On Automatic Control - 1994 - VOL.39 - NO. 2 - pp. 315-328;

[10]Gantmaher F.R. Teoriya matrits/ F.R.Gantmaher -4-e izd.- M.: Nauka, 1988.- 552s.

\section{ИССЛЕДОВАНИЕ ЭНЕРГЕТИЧЕСКИХ ХАРАКТРЕИСТИК КОГЕНЕРАЦИОННОЙ ЭНЕРГЕТИЧЕСКОЙ УСТАНОВКИ В УСЛОВИЯХ ИЗМЕНЕНИЯ КАЧЕСТВА ТОПЛИВА}

The research of the energy characteristics of cogeneration power plant in a changing fuel quality

к.т.н., зав. лаб., Тарахтий О.С. (Tarakhtii O.S.), Бундюк А.Н. (Bundiuk A.N.), к.т.н., профессор

Одесский национальный политехнический университет, Одесса, E-mail: larionova-olya@ mail.ru

Copyright (C) 2014 by author and the journal “Automation technological and business - processes".

This work is licensed under the Creative Commons Attribution International License (CC BY).

http://creativecommons.org/licenses/by/4.0/

\section{Аннотация}

Из-за несовершенства технологических производств многие промышленные предприятия имеют побочные газообразные продукты, которые относятся к вторичным энергоресурсам. Использование таких газов в качестве топлива для энергетических установок данных предприятий является актуальной задачей энергосбережения. В данной статье рассматривается возможность использования технологических газов и газов искусственного происхождения в качестве топлива для когенерационной энергетической установки на базе газотурбинного двигателя. Использование данных видов топлива в когенерационны энергетических установках позволит снизить стоимость выпускаемой продукции за счет снижения затрат на топливо. Однако использование подобных газов в качестве основного топлива для энергоустановок

осложняется тем, что низшая теплота сгорания таких газов может существенно изменяться и, таким образом вносить существенные возмущения в работу энергетических установок. Проведен анализ влияния изменения низшей теплоты сгорания рассматриваемых газов на энергетические характеристики теплового двигателя когенерационной энергетической установки и определены допустимые пределы изменения низшей теплоты сгорания топлива. Предложены структура системы автоматического регулирования и метод управления мощностью газотурбинной установки за счет перераспределения потоков первичного и вторичного воздуха в камере сгорания. В контур регулирования температуры газов на выходе из камеры сгорания газотурбинной $\backslash$ 